\title{
REMARKS ON DOMAIN-WALL FERMIONS
}

\author{
MICHAEL CREUTZ \\ Physics Department \\ Brookhaven National Laboratory \\ Upton, NY 11973 \\ $U S A$ \\ creutz@bnl.gov
}

\section{Introduction}

Issues of chiral symmetry permeate theoretical physics. Our understanding of pionic interactions revolves around spontaneous symmetry breaking and approximately conserved axial currents. The standard model itself is truly chiral, with the weak gauge bosons only coupling to one helicity state of the fundamental fermions. In the context of unification, chiral symmetry provides a mechanism for protecting fermion masses, possibly explaining how a theory at a much higher scale can avoid large renormalizations of the light particle masses. Extending this mechanism to bosons provides one of the more compelling motivations for super-symmetry.

On the lattice, chiral symmetry raises many interesting issues. These are intricately entwined with the famous axial anomalies and the so called "doubling" problem. Being a full regulator, the lattice must break some aspects of chiral symmetry to give the required anomalies in the continuum limit. Prescriptions for lattice fermions that do not accommodate anomalies cancel them with spurious extra species (doublers). Domain-wall fermions[1], the motivation for this talk and the subject of most of todays presentations, are one scheme to minimize these necessary symmetry violations.

But speak to an audience that already knows all this. In an attempt to avoid boring you, I will discuss domain-wall fermions from a rather unconventional direction. Following a recent paper of mine [2], I present the subject from a "chemists" point of view, in terms of a chain molecule with special electronic states carrying energies fixed by symmetries. For lattice gauge theory, placing one of these molecules at each space-time site gives excitations of naturally zero mass. This is in direct analogy to the role of 
chiral symmetry in conventional continuum descriptions. After presenting this picture, I will wander into some comments and speculations about exact lattice chiral symmetries and schemes for gauging them.

\section{A ladder molecule}

To start, let me consider two rows of atoms connected by horizontal and diagonal bonds, as illustrated here

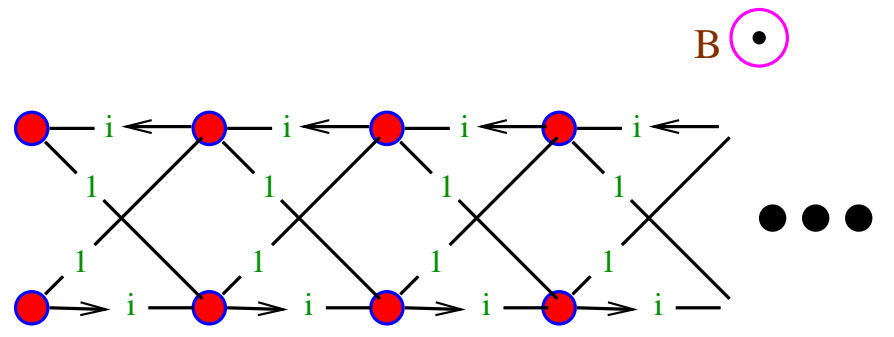

The bonds represent hopping terms, wherein an electron moves from one site to another via a creation-annihilation operator pair in the Hamiltonian. Later I will include vertical bonds, but for now consider just the horizontal and diagonal connections.

Years ago during a course on quantum mechanics, I heard Feynman present an amusing description of an electron's behavior when inserted into a lattice. If you place it initially on a single atom, the wave function will gradually spread through the lattice, much like water poured in a cell of a metal ice cube tray. With damping, it settles into the ground state which has equal amplitude on each atom. To this day I cannot fill an ice cube tray without thinking of this analogy and pouring all the incoming water into a single cell.

I now complicate this picture with a magnetic field applied orthogonal to the plane of the system. This introduces phases as the electron hops, causing interesting interference effects. In particular, consider a field of onehalf flux unit per plaquette. This means that when a particle hops around a unit area (in terms of the basic lattice spacing) the wave function picks up a minus sign. Just where the phases appear is a gauge dependent convention; only the total phase around a closed loop is physical. One choice for these phases is indicated by the numbers on the bonds in the above picture.

The phase factors cause cancellations and slow diffusion. For example, consider the two shortest paths between the sites $\mathbf{a}$ and $\mathbf{b}$ in the following picture 


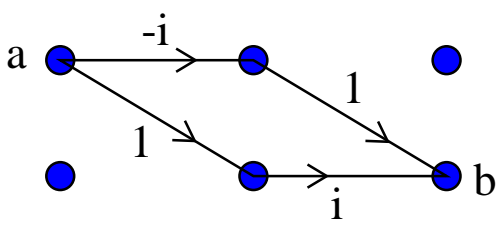

With the chosen flux, these paths exactly cancel. For the full molecule this cancellation extends to all paths between these sites. An electron placed on site a can never diffuse to site b. Unlike in the ice tray analogy, the wave function will not spread to any site beyond the five nearest neighbors.

As a consequence, the Hamiltonian has localized eigenstates. While it is perhaps a bit of a misuse of the term, these states are "soliton-like" in that they just sit there and do not change their shape. There are two such states per plaquette; one possible representation for these two states is shown in the following figure

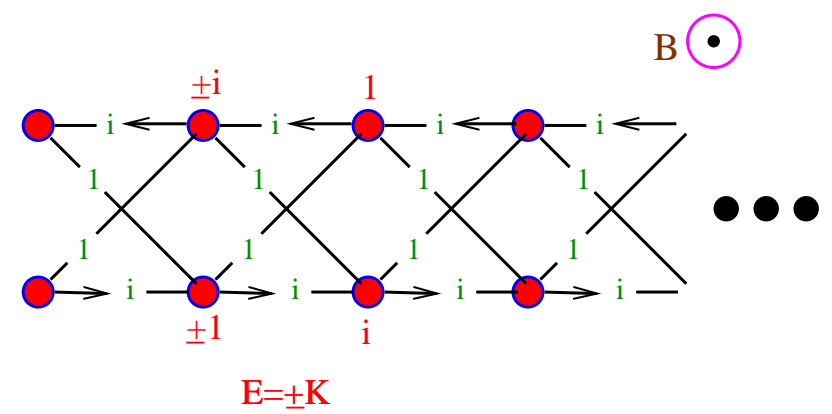

The states are restricted to the four sights labeled by their relative wave functions. Their energies are fixed by the size of the hopping parameter $K$.

For a finite chain of length $L$ there are $2 L$ atoms, and thus there should be a total of $2 L$ possible states for our electron (ignoring spin for the moment). There are $L-1$ plaquettes, and thus $2 L-2$ of the above soliton states. This is almost the entire spectrum of the Hamiltonian, but two states are left over. These are zero energy states bound to the ends of the system. The wave function for one of those is shown here

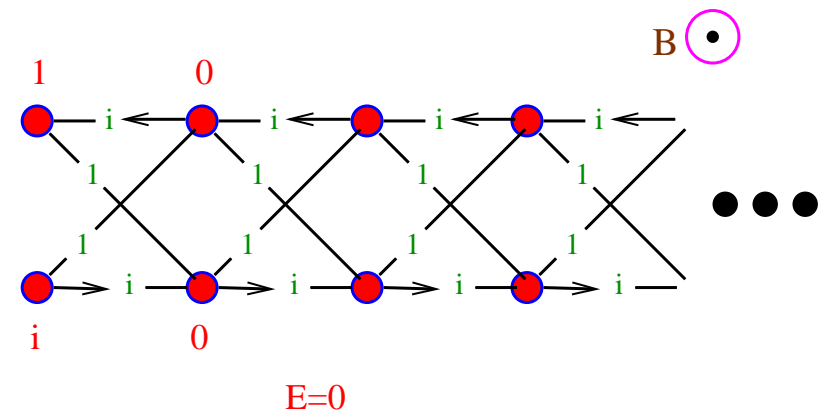


We now have the full spectrum of the Hamiltonian: $L-1$ degenerate states of positive energy, a similar number of degenerate negative energy states, and two states of zero energy bound on the ends.

Now consider what happens when vertical bonds are included in our molecule. The phase cancellations are no longer complete and the solitonic states spread to form two bands, one with positive and one with negative energy. However, for our purposes, the remarkable result is that the zero modes bound on the ends of the chain are robust. The corresponding wave functions are no longer exactly located on the last atomic pair, but now have an exponentially suppressed penetration into the chain. The following figure shows the wave function for one of these states when the vertical bond has the same strength as the others. There is a corresponding state on the other end of the molecule.

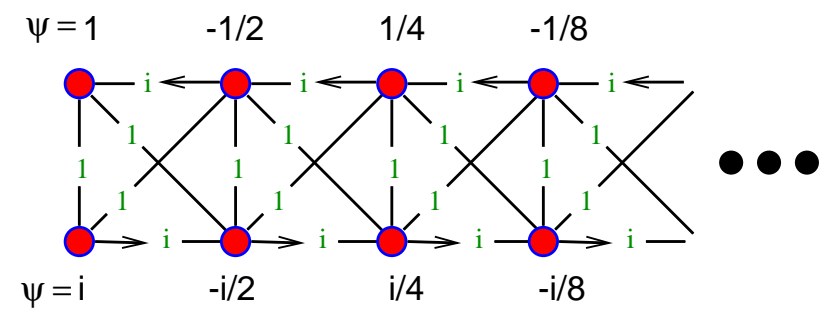

When the chain is very long, both of the end states are forced to zero energy by symmetry considerations. First, since nothing distinguishes one end of the chain from the other, they must have equal energy, $E_{L}=E_{R}$. On the other hand, a change in phase conventions, effectively a gauge change, can change the sign of all the vertical and diagonal bonds. Following this with a left right flip of the molecule will change the signs of the horizontal bonds. This takes the Hamiltonian to its negative, and shows that the states must have opposite energies, $E_{L}=-E_{R}$. This is indicative of a particlehole symmetry. The combination of these results forces the end states to zero energy, with no fine tuning of parameters.

For a finite chain, the exponentially decreasing penetration of the end states into the molecule induces a small interaction between them. They mix slightly to acquire exponentially small energies $E \sim \pm e^{-\alpha L}$. As the strength of the vertical bonds increases, so does the penetration of the end states. At a critical strength, the mixing becomes sufficient that the zero modes blend into the positive and negative energy bands. In the full model, the mixing depends on the physical momentum, and this disappearance of the zero modes is the mechanism that removes the "doublers" when spatial momentum components are near $\pi$ in lattice units [3].

Energy levels forced to zero by symmetry lie at the core of the domain wall fermion idea. On every spatial site of a three dimensional lattice we place one of these chain molecules. The distance along the chain is usually 
referred to as a fictitious "fifth" dimension. The different spatial sites are coupled, allowing particles in the zero modes to move around. These are the physical fermions. The symmetries that protect the zero modes now protect the masses of these particles. Their masses receive no additive renormalization, exactly the consequence of chiral symmetry in the continuum. The physical picture is sketched in this cartoon

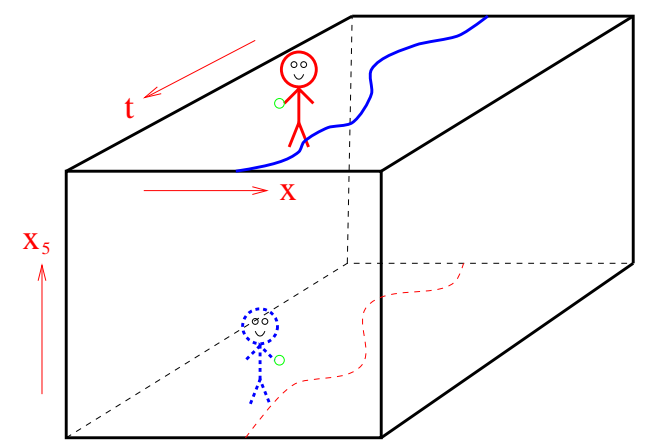

where I have rotated the fifth dimension to the vertical. Our world lines traverse the four dimensional surface of this five dimensional manifold.

Actually, the connection with chiral symmetry is much deeper than just an analogy. The construction guarantees that the modes are are automatically chiral. To see how this works, place Pauli spin matrices on the spatial bonds. This couples the phases seen by the particles to their spins. The zero mode that is attracted to one end of the chain will continue to move spatially in a direction corresponding to its helicity, as sketched here

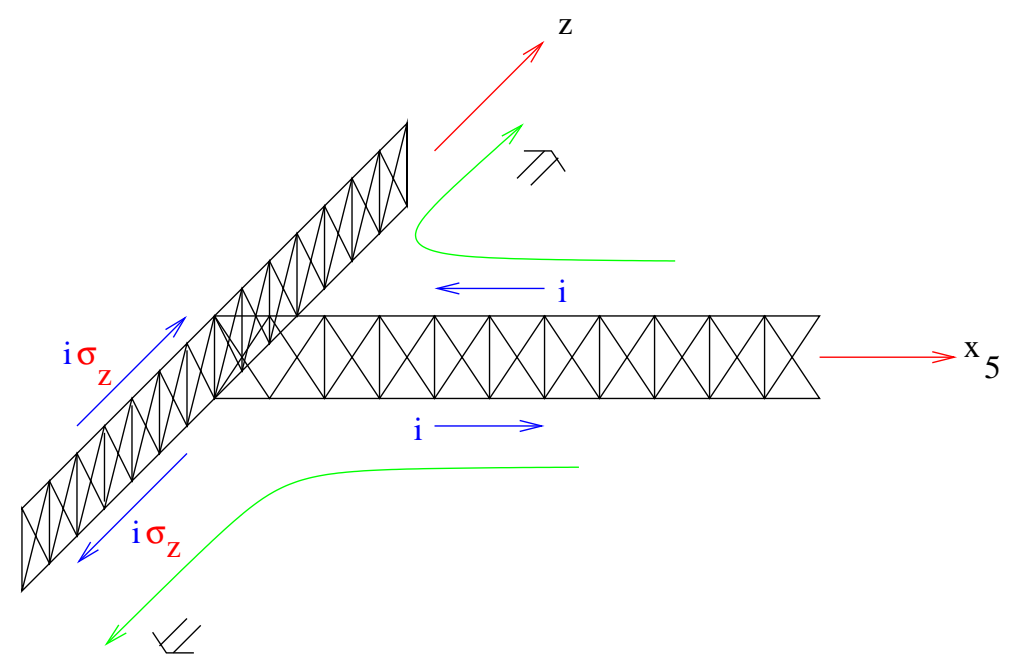

The "device" in this figure is effectively a helicity projector. This construc- 
tion is equivalent to choosing a particular set of Dirac gamma matrices

$$
\begin{gathered}
\gamma_{0}=\left(\begin{array}{ll}
0 & 1 \\
1 & 0
\end{array}\right) \\
\gamma_{5}=\left(\begin{array}{cc}
0 & -i \\
i & 0
\end{array}\right) \\
\gamma_{i}=\left(\begin{array}{cc}
0 & -i \sigma_{i} \\
i \sigma_{i} & 0
\end{array}\right)
\end{gathered}
$$

\section{Slicing the fifth dimension}

I hope this description of domain-wall fermions in terms of simple chain molecules has at least been thought provoking. I now ramble on with some general remarks about the basic scheme. The existence of the end states relies on using open boundary conditions in the fifth direction. If we were to curl our extra dimension into a circle, they will be lost. To retrieve them, consider cutting such a circle, as in this figure

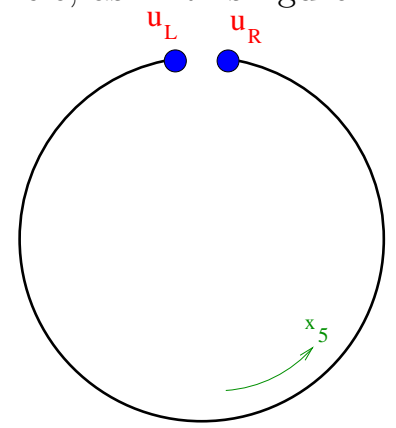

Of course, if the size of the extra dimension is finite, the modes mix slightly. This is crucial for the scheme to accommodate anomalies [3].

Suppose I want a theory with two flavors of light fermion, such as the up and down quarks. For this one might cut the circle twice, as shown here

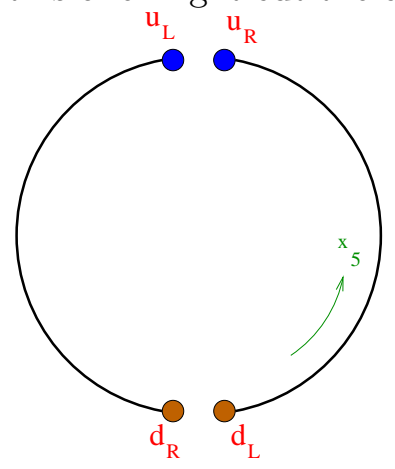

Remarkably, this construction keeps one chiral symmetry exact, even if the size of the fifth dimension is finite. Since the cutting divides the molecule 
into two completely disconnected pieces, in the notation of the figure we have the number of $u_{L}+d_{R}$ particles absolutely conserved. Similarly with $u_{R}+d_{L}$. Subtracting, we discover an exactly conserved axial charge corresponding to the continuum current

$$
j_{\mu 5}^{3}=\bar{\psi} \gamma_{\mu} \gamma_{5} \tau^{3} \psi
$$

The conservation holds even with finite $L_{5}$. There is a small flavor breaking since the $u_{L}$ mixes with the $d_{R}$. These symmetries are reminiscent of KogutSusskind [4], or staggered, fermions, where a single exact chiral symmetry is accompanied by a small flavor breaking. Now, however, the extra dimension gives additional control over the latter.

Despite this analogy, the situation is physically somewhat different in the zero applied mass limit. Staggered fermions are expected to give rise to a single zero mass Goldstone pion, with the other pions acquiring mass through the flavor breaking terms. In my double cut domain-wall picture, however, the zero mass limit has three degenerate equal mass particles as the lowest states. To see how this works it is simplest to discuss the physics in a chiral Lagrangian language. The finite fifth dimension generates an effective mass term, but it is not in a flavor singlet direction. Indeed, it is in a flavor direction orthogonal to the naive applied mass. In the usual "sombrero" picture of the effective Lagrangian, as illustrated here,

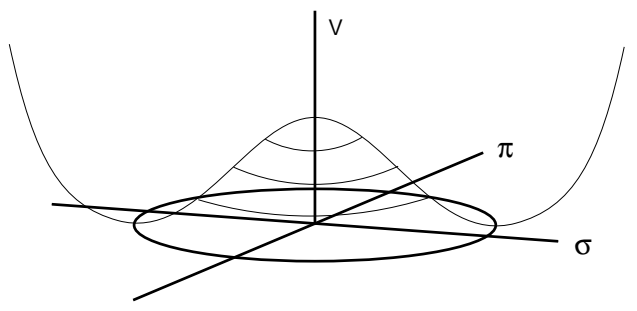

the two mass terms compete and the true vacuum rotates around the Mexican hat from the conventional "sigma" direction to the "pi" direction.

\section{How many fermions?}

Now I become more speculative. The idea of cutting multiply the fifth dimension to obtain several species suggests extensions to zero modes on more complicated manifolds. By having multiple zero modes, we have a mechanism to generate multiple flavors. Maybe one can have a theory where all the physical fermions in four dimensions arise from a single fermion field in the underlying higher dimensional theory. Schematically we might have something like 


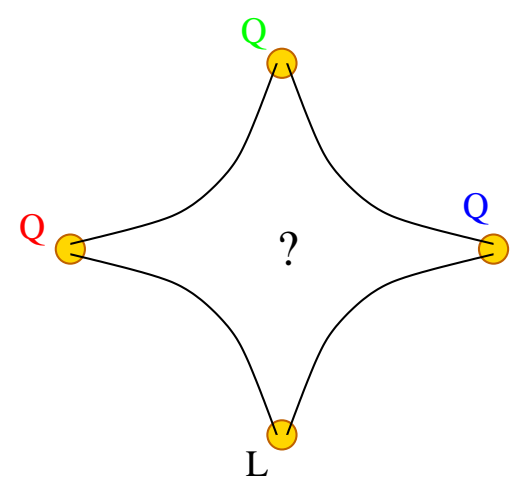

where each point represents some four dimensional surface and the question remark represents structures in the higher dimension that need specification.

One nice feature provided by such a scheme is a possible mechanism for the transfer of various quantum numbers involved in anomalous processes. For example, the baryon non-conserving 't Hooft process[5] might arise from a lepton flavor tunneling into the higher manifold and reappearing on another surface as a baryon.

I've been rather abstract here. This generic mechanism is in fact the basis of one specific proposed formulation of the standard model on the lattice[6]. For this model the question mark in the above figure is a fourfermi interaction in the interior of the extra dimension, as sketched here

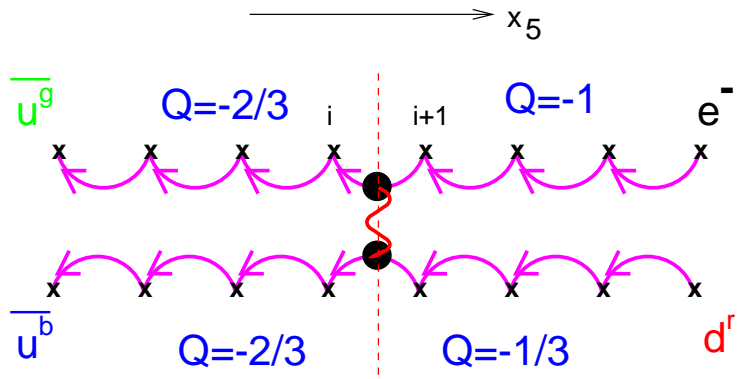

In some sense, the right-handed doubler of the left-handed electron is interpreted as an anti-quark. This picture appears to have all the necessary ingredients for a fully regulated and exactly gauge invariant formulation of the standard model. The primary uncertainty lies with side effects of the multiple fermion coupling. Our experience with non-perturbative phenomena involving strongly coupled fermions is rather limited; in particular, the model cannot tolerate the generation of a spontaneous symmetry breaking of any of the gauge symmetries at the scale of the cutoff. 


\section{Summary}

I have presented a simple molecular picture for zero modes protected by symmetry. This illustrates the mechanism for mass protection in the domainwall formulation of lattice fermions. Then I discussed how some chiral symmetries can become exact in this approach. Finally I speculated on schemes for generating multiple fermion species from the geometry of higher dimensional models. The latter may have connections with the activities in string theory.

\section{Acknowledgment}

This manuscript has been authored under contract number DE-AC0298CH10886 with the U.S. Department of Energy. Accordingly, the U.S. Government retains a non-exclusive, royalty-free license to publish or reproduce the published form of this contribution, or allow others to do so, for U.S. Government purposes.

\section{References}

1. D. Kaplan, Phys. Lett. B288 (1992) 342; V. Furman and Y. Shamir, Nucl. Phys. B439, 54 (1995).

2. M. Creutz, Phys. Rev. Lett. 83, 2636 (1999).

3. M. Creutz and I. Horvath, Phys. Rev. D50, 2297 (1994); Nucl. Phys. B34 (Proc. Suppl.), 586 (1994).

4. J. Kogut and L. Susskind, Phys. Rev. D11, 395 (1975).

5. G. t'Hooft, Phys. Rev. Lett. 37, 8 (1976); Phys. Rev. D14, 3432 (1976).

6. M. Creutz, C. Rebbi, M. Tytgat, S.-S. Xue, Physics Letters B402, 341-345 (1997); M. Creutz, Nuclear Physics B (Proc.Suppl.) 63A-C, 599 (1998). 